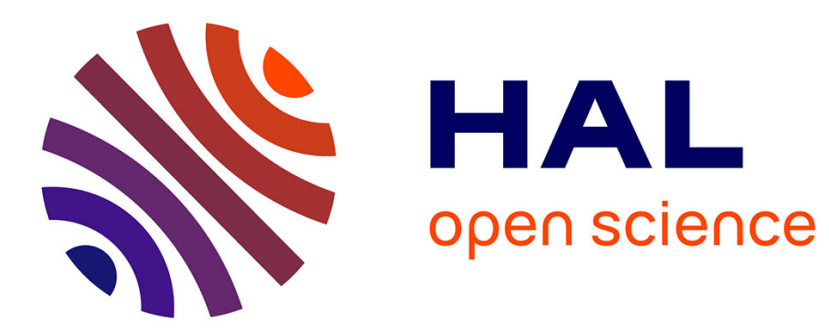

\title{
The structure of a Burkholderia pseudomallei immunophilin-inhibitor complex reveals new approaches to antimicrobial development
}

Isobel H Norville, Katherine O’Shea, Mitali Sarkar-Tyson, Suxin Zheng, Richard W Titball, Gabriele Varani, Nicholas J Harmer

\section{To cite this version:}

Isobel H Norville, Katherine O'Shea, Mitali Sarkar-Tyson, Suxin Zheng, Richard W Titball, et al.. The structure of a Burkholderia pseudomallei immunophilin-inhibitor complex reveals new approaches to antimicrobial development. Biochemical Journal, 2011, 437 (3), pp.413-422. 10.1042/BJ20110345 . hal-00608383

\section{HAL Id: hal-00608383 \\ https://hal.science/hal-00608383}

Submitted on 13 Jul 2011

HAL is a multi-disciplinary open access archive for the deposit and dissemination of scientific research documents, whether they are published or not. The documents may come from teaching and research institutions in France or abroad, or from public or private research centers.
L'archive ouverte pluridisciplinaire HAL, est destinée au dépôt et à la diffusion de documents scientifiques de niveau recherche, publiés ou non, émanant des établissements d'enseignement et de recherche français ou étrangers, des laboratoires publics ou privés. 
The structure of a Burkholderia pseudomallei immunophilin-inhibitor complex reveals new approaches to antimicrobial development

Isobel H. Norville ${ }^{*}$, , Katherine O’Shea ${ }^{*}$, , Mitali Sarkar-Tyson $^{\dagger}$, Suxin Zheng ${ }^{\ddagger}$, Richard W. Titball $^{*}$, Gabriele Varani ${ }^{\ddagger}, \S$ and Nicholas J. Harmer ${ }^{*} \|$.

* School of Biosciences, University of Exeter, Geoffrey Pope Building, Stocker Road, Exeter EX4 4QD, U.K.

${ }^{\dagger}$ Defence Science and Technology Laboratory, Porton Down, Salisbury, Wiltshire SP4 0JQ, U.K.

${ }^{\ddagger}$ Department of Chemistry and Department of Biochemistry, University of Washington, Box 351700, Seattle, WA 98195-1700, U.S.A.

Addresses for correspondence:

$\S$ Department of Chemistry, University of Washington, Box 351700, Seattle, WA 981951700, U.S.A.

e-mail: varani@ chem.washington.edu

Tel: +01 206 543-7113

Fax: +01 206 685-8665

॥ School of Biosciences, University of Exeter, Geoffrey Pope Building, Stocker Road, Exeter EX4 4QD, U.K.

e-mail: N.J.Harmer@exeter.ac.uk.

Tel: +44 (0) 1392725179

Fax: +44 (0) 1392263489

Running Title: Structure of a Burkholderia pseudomallei immunophilin-inhibitor complex

Keywords: Prolyl-peptide isomerase, Burkholderia pseudomallei, X-ray crystallography, NMR, small molecule inhibitor.

Abbreviations: Burkholderia pseudomallei Mip-like protein, BpML1; FK506 binding protein, FKBP; heteronuclear single-quantum coherence, HSQC; Legionella pneumophila Mip, LpMip; Macrophage infectivity potentiator, Mip; Prolyl peptide isomerase, PPIase; threedimensional, 3D. 


\section{Synopsis}

Macrophage infectivity potentiators (Mips) are a subset of immunophilins associated with virulence in a range of micro-organisms. These proteins possess prolyl-peptide isomerase activity and are inhibited by drugs including rapamycin and tacrolimus. We determined the structure of the Mip homologue (BpML1) from the human pathogen and biowarfare threat Burkholderia pseudomallei by nuclear magnetic resonance and X-ray crystallography. The crystal structure suggests that key catalytic residues in the BpML1 active site have

unexpected conformational flexibility consistent with a role in catalysis. The structure further revealed BpML1 binding to a helical peptide, in a manner resembling the physiological interaction of human TGF $\beta$ receptor 1 with the human immunophilin FKBP12. Furthermore, the structure of BpML1 bound to the class inhibitor cycloheximide $\mathrm{N}$-ethylethanoate showed that this inhibitor mimics such a helical peptide, in contrast to the extended prolyl peptide mimicking shown by inhibitors such as tacrolimus. We suggest that Mips, and potentially other bacterial immunophilins, participate in protein-protein interactions in addition to their prolyl-peptide isomerase activity, and that some roles of Mip proteins in virulence are independent of their prolyl-peptide isomerase activity. 


\section{Introduction}

Melioidosis is a community acquired infection frequent in South-East Asia and Northern Australia [1]. Caused by the Gram-negative bacterium Burkholderia pseudomallei, the disease can range from a localised infection to an acute systemic, chronic or persistent disease. The symptoms of infection are diverse, often resembling tuberculosis or cancer. Consequently, the disease is likely to be often misdiagnosed [2]. The disease is especially pernicious as the bacterium is intrinsically resistant to a wide range of antibiotics [3]: even with optimal treatment, mortality in acute cases of disease ranges from 20-40\% [2]. B. pseudomallei is also a potential bioterror agent [4]. Consequently, there has been a recent and concerted effort to develop novel countermeasures against this organism.

One potential target class for novel antimicrobials is the FK506 (tacrolimus) binding proteins. These ubiquitous enzymes catalyse the isomerisation of pre-proline peptide bonds between the cis and trans configurations (peptidyl-prolyl isomerase, or PPIase, activity [5]), and form part of the broader category of PPIase enzymes. Several FKBPs have been found to play a role in virulence in a range of species including the bacteria Legionella pneumophila [6], Chlamydia trachomatis [7], Neisseria gonorrhoeae [8] and the protozoan Trypanosoma cruzi [9]. One of the first bacterial FKBPs to be studied in detail is a L. pneumophila protein required for the efficient invasion of macrophages [6]. Consequently, these proteins were labelled Mip (macrophage infectivity potentiator) proteins. The FKBP domain of the Legionella Mip (LpMip) is required for virulence, and both antibodies binding to the active site and specific inhibitors sufficed to reduce the capacity of Legionella to invade macrophages $[10,11]$. We recently showed that a Mip-like protein from B. pseudomallei, BPSS1823 (hereafter BpML1), has high PPIase activity; and that it is required for efficient invasion of host cells and virulence in a mouse model of infection. Deletion of this gene significantly attenuates B. pseudomallei (Norville, I., Harmer, N., Harding, S., Fischer, G., Keith, K., Brown, K., Sarkar-Tyson, M., Titball, R., unpublished work).

Despite extensive study of Mips in a range of microorganisms, the true biological targets for these proteins have not been clearly elucidated $[8,11,12]$. It has been broadly assumed that they act solely as isomerases [11]. Mips have been suggested to be an attractive target class for antimicrobials: there are known high affinity inhibitors, indicating that the proteins are eminently druggable; and their prokaryotic functions are differentiated from those of higher eukaryotic FKBPs.

We have previously shown that the B. pseudomallei Mip-like protein plays a key role in disease progression. In this study, we report the molecular structure of BpML1, identify common features with other Mips, and highlight differences from mammalian FKBPs that could be exploited for drug design. We also identify new approaches to the design of compounds able to block this target. These compounds could be developed as antimicrobials to treat melioidosis, but could also have a much wider utility to treat other diseases where Mips play a key role in infection. 


\section{Methods \\ Preparation of BpML1}

Full length BpML1 from B. pseudomallei strain K96243 was cloned into pET15b using the NdeI and HindIII sites. The vector was transformed into BL21 (DE3) cells. For enzymology, cells were grown in $\mathrm{LB}$ media at $37^{\circ} \mathrm{C}$ with agitation until the $\mathrm{OD}_{600}$ reached 0.4-0.6.

Isopropyl $\beta$-D-1-thiogalactopyranoside was added to $1 \mathrm{mM}$ and growth continued at $20^{\circ} \mathrm{C}$ for 4 hours. Harvested cells were resuspended in $10 \mathrm{mM}$ PBS supplemented with $100 \mathrm{mg} / \mathrm{mL}$ DNase I and Complete EDTA-free protease inhibitors (Roche), and disrupted by sonication. Clarified lysate was loaded onto a $1 \mathrm{ml}$ Histrap FF column (GE Healthcare) and the recombinant protein eluted in $10 \mathrm{mM}$ PBS supplemented with $100 \mathrm{mM}$ imidazole. The purified protein was dialysed against $10 \mathrm{mM}$ PBS and samples frozen at $-80^{\circ} \mathrm{C}$ until use. Protein concentration was determined using a bicinchoninic acid assay (Pierce Biotechnology).

For crystallisation, cells were grown in ZYM-5052 media [13] supplemented with $100 \mu \mathrm{g} / \mathrm{mL}$ ampicillin at $37^{\circ} \mathrm{C}$ until the $\mathrm{OD}_{600}$ was 0.5 , and then at $20^{\circ} \mathrm{C}$ for 16 hours. Harvested cells were resuspended in $20 \mathrm{mM}$ Tris- $\mathrm{HCl} \mathrm{pH} 8.0,0.5 \mathrm{M} \mathrm{NaCl}$ (Buffer A), and lysed using a Soniprep 150 sonicator (MSE). Clarified lysate was purified using a nickel-agarose column (Bioline). Briefly, the loaded protein was washed with Buffer A supplemented with $20 \mathrm{mM}$ imidazole- $\mathrm{HCl} \mathrm{pH} \mathrm{8.0,} \mathrm{and} \mathrm{eluted} \mathrm{with} \mathrm{Buffer} \mathrm{A} \mathrm{supplemented} \mathrm{with} 250 \mathrm{mM}$ imidazole-HCl pH 8.0. BpML1 was then loaded onto a Superdex $20016 / 60 \mathrm{hr}$ column (GE Healthcare), and eluted isocratically with $10 \mathrm{mM}$ Hepes pH 7.0, $0.5 \mathrm{M} \mathrm{NaCl}$.

For NMR studies, ${ }^{15} \mathrm{~N}$ labelled and ${ }^{13} \mathrm{C}^{15} \mathrm{~N}$ labelled protein was made by substituting $\left[{ }^{15} \mathrm{~N}\right] \mathrm{NH}_{4} \mathrm{Cl}$ and/or $\left[{ }^{13} \mathrm{C}\right]$ glucose in the cell growth media. ${ }^{15} \mathrm{~N}$ labelled and ${ }^{13} \mathrm{C}^{15} \mathrm{~N}$ double labelled proteins were dialysed into $20 \mathrm{mM}$ potassium phosphate $\mathrm{pH} 7.0,100 \mathrm{mM} \mathrm{KCl}, 2 \mathrm{mM}$ DTT, to a final protein concentration of approximately $1 \mathrm{mM}$. Cycloheximide $\mathrm{N}$ ethylethanoate (>95\%) was purchased from Life Chemicals (www.lifechemicals.com) without further purification. Samples of the complex were prepared by titrating unlabeled cycloheximide N-ethylethanoate into labelled protein samples while monitoring ${ }^{15} \mathrm{~N}$ heteronuclear single-quantum coherence (HSQC) chemical shift change until a previously established "saturated" spectrum was obtained.

\section{Crystallisation and structure solution}

All crystals were grown using the microbatch method, and were prepared using an Oryx6 crystallisation robot (Douglas Instruments). BpML1 at $14 \mathrm{mg} / \mathrm{mL}$ was mixed with an equal volume of $2.2 \mathrm{M}\left(\mathrm{NH}_{4}\right)_{2} \mathrm{SO}_{4}, 0.1 \mathrm{M}$ Bis-Tris $\mathrm{pH} 5.5$, and grown at $20^{\circ} \mathrm{C}$. Prior to flashfreezing in liquid nitrogen, crystals were soaked for 30-60 seconds in a cryoprotectant solution of $1.1 \mathrm{M}\left(\mathrm{NH}_{4}\right)_{2} \mathrm{SO}_{4}, 0.1 \mathrm{M}$ Bis-Tris $\mathrm{pH} 5.5,30 \%$ (v/v) glycerol. Single wavelength X-ray diffraction data were collected at a wavelength of $0.861 \AA$ at $100 \mathrm{~K}$ at beamline I03 of the Diamond synchrotron (Table 1). Data were processed using iMOSFLM version 1.0.3 [14] and SCALA [15]. Initial phases were determined by molecular replacement using the structures of human FKBP12 (PDB ID: 1FKB) and the N-terminal domain of rabbit FKBP59 (PDB ID: 1ROT) as a model. These structures were modified to remove non-homologous side chains using CHAINSAW [16]. Molecular replacement was performed using PHASER [17]. One protomer was observed in the asymmetric unit. Model building and refinement of the structures was performed using Coot version 0.6.1 [18] and PHENIX version 1.6.1 [19]. The structure was solved using anisotropic B-factors for all heavy atoms, riding hydrogen atoms, and optimisation of stereochemical and ADP restraints. Structures were validated using PHENIX, Coot and MOLPROBITY [20]. The Ramachandran plot for the final model showed $100 \%$ of residues in the favoured region. Structural images were prepared using the PyMOL molecular graphics system, version 1.3 (Schrödinger). 


\section{NMR structure solution}

All NMR spectra were collected at $298 \mathrm{~K} .{ }^{15} \mathrm{~N}$ HSQC, triple-resonance spectroscopy, HCCH TOCSY [21], three-dimensional (3D) ${ }^{15} \mathrm{~N}$-edited NOESY and 3D ${ }^{13} \mathrm{C}$-edited NOESY [22] were recorded on a Bruker Avance 500-MHz spectrometer with cryoprobe. The $2 \mathrm{D}{ }^{1} \mathrm{H}-{ }^{1} \mathrm{H}$ NOESY and ${ }^{1} \mathrm{H}-{ }^{1} \mathrm{H}$ TOCSY spectra were recorded on a Bruker Avance $750-\mathrm{MHz}$ spectrometer. Backbone resonance assignments of $\mathrm{H}, \mathrm{N}, \mathrm{C}_{\alpha}, \mathrm{C}^{\prime}$, and side-chain $\mathrm{C}_{\beta}$ were performed using triple-resonance HNCA [23], $\mathrm{CBCA}(\mathrm{CO}) \mathrm{NH}$ [24], HNCACB, and $\mathrm{HN}(\mathrm{CO}) \mathrm{CA}$. The remaining side-chain resonance assignments were obtained from the analysis of ${ }^{15} \mathrm{~N}$-edited NOESY, ${ }^{13} \mathrm{C}$-edited NOESY, and HCCH-TOCSY spectra. Aromatic ring protons and protected amides were identified from two-dimensional ${ }^{1} \mathrm{H}^{-1} \mathrm{H}$ NOESY, ${ }^{1} \mathrm{H}-$ ${ }^{1} \mathrm{H}$ TOCSY, and ${ }^{15} \mathrm{~N}$ HSQC, respectively, collected from a sample exchanged into a $100 \%$ ${ }^{2} \mathrm{H}_{2} \mathrm{O}$ NMR buffer. The assignments of the ligand were obtained based on the $2 \mathrm{D}{ }^{13} \mathrm{C}$-filtered NOESY and total correlation spectroscopy (TOCSY) spectra of the "F1fF2f" type [25]. The $2 \mathrm{D}{ }^{13} \mathrm{C}-{ }^{12} \mathrm{C}$ NOESY spectra were collected to facilitate the assignments of protein-ligand intermolecular NOEs [26].

Spectral data were processed using NMRPipe [27] and analyzed using CcpNmr Analysis1.0. [28]. The structure calculations were conducted automaticaliy by CYANA2.1 [29, 30] based on nuclear Overhauser enhancements (NOEs) selected from ${ }^{15} \mathrm{~N}$-edited NOE spectroscopy (NOESY), ${ }^{13} \mathrm{C}$-edited NOESY, ${ }^{1} \mathrm{H}-{ }^{1} \mathrm{H}$ NOESY and ${ }^{13} \mathrm{C}-{ }^{12} \mathrm{C}$ NOESY, as well as additional dihedral-angle and hydrogen-bond constraints generated from the NMR data.

\section{Inhibitor assay}

PPIase activity of BpML1 protein was determined by a protease coupled assay as described previously [31]. Briefly, $10 \mathrm{nM} \mathrm{BpML1}$ protein was incubated for 6 min at $6^{\circ} \mathrm{C}$ in $1.2 \mathrm{~mL} 35$ $\mathrm{mM}$ Hepes buffer, $\mathrm{pH} 7.8$ with succinyl-Ala-Phe-Pro-Phe- $p$-nitroanilide $(10 \mathrm{mg} / \mathrm{mL}$; Bachem). $\alpha$-chymotrypsin (Sigma) was added to the cuvette to a final concentration of $1.25 \mathrm{mg} / \mathrm{mL}$ and mixed. Hydrolysis of the substrate was measured at $390 \mathrm{~nm}$ using a Shimadzu $1800 \mathrm{UV} / \mathrm{V}$ is spectrophotometer at $1 \mathrm{sec}$ intervals until there was no further change in absorbance. For inhibition measurements, BpML1 protein was pre-incubated with varying concentrations of cycloheximide-N-ethylethanoate from $1-500 \mu \mathrm{M}$ for 6 min prior to addition of chymotrypsin. At least three independent readings were taken at each data point. All data fitting and statistical analyses were performed using SPSS v16.0 (IBM). The pseudo first order rate constant was calculated using equation 1 [32]; data from 10-50 sec (following the lag phase, and before substrate became limiting) were taken, and $\mathrm{k}_{\mathrm{obs}}$ calculated by linear regression.

(1) $\ln \left[A_{\infty}-A_{\mathrm{t}}\right]=-k_{\mathrm{obs}} t+\ln \left[A_{\infty}-A_{0}\right]$

The enzymatic rate was determined by comparing the observed rate to the uncatalysed rate (equation 2).

(2) $k_{\text {enz }}=k_{\text {obs }}-k_{\text {uneat }}$

Data for inhibitor assays were fit to equation 3 [33] using least squares non-linear fitting. [E] was treated as a constant $(10 \mathrm{nM}) ; \mathrm{v}_{0}$ and $K_{\mathrm{I}}^{\text {app }}$ were fit, using initial estimates based on the raw data.

(3) $v=v_{0} \frac{[E]^{-}[I]^{-} K+\sqrt{\left([E]^{-}[I]^{-} K\right)^{2}+4[E] K}}{2[E]}$ 


\section{Results and Discussion}

\section{The BpML1 fold and active site residue configuration are highly similar to LpMip}

We solved the X-ray structure of BpML1 to $0.91 \AA$ (Table 1). The structure confirmed that BpML1 adopts a classical FKBP fold (Figure 1A). Closer inspection of the active site in the crystal revealed that key side chains adopt a conformation that closely resembles that of the ligand free L. pneumophila Mip FKBP domain (Figure 1B). NMR structures of BpML1 that were determined independently show an excellent similarity in overall fold with the crystal structure. They also demonstrate that there is considerable flexibility in both peripheral loops and side chains (Figure 1C) in the well defined active site (see Supplementary Figure 1 online $[34,35])$. In particular, the $\beta 4-\beta 5$ loop, which contributes the key $\operatorname{Tyr}^{89}$ side chain to the active site, shows two distinct conformations in solution; and several of the key conserved active site side chains have considerable flexibility. These observations are in contrast to Mip from L. pneumophila and T. cruzi, which show a remarkable commonality in the conformations adopted by these regions (see Supplementary Figure 2 online [34, 36, 37]). These observations suggest that previous structures (especially crystal structures) might be trapping a preferred, low energy conformation that may be catalytically relevant, but which does not capture the full range of dynamics available to the enzyme in solution.

\section{BpML1 forms protein-protein interactions reminiscent of other FKBPs}

The protein sample used for crystallisation of BpML1 had been cloned with a $6 *$ His tag, linked to the protein by a thrombin cleavage site (sequence

MSSHHHHHHSSGLVPRGSHM..., thrombin site underlined). The thrombin cleavage peptide was clearly resolved in the electron density, and forms an interaction with a neighbouring molecule in the crystal. This interaction placed the peptide directly in the active site of the neighbouring molecule, forming an apparently tight interaction, burying $890 \AA^{2}$ of surface (Figure 2A). Intriguingly, although BpML1 is a very active PPIase (Norville, I., Harmer, N., Harding, S., Fischer, G., Keith, K., Brown, K., Sarkar-Tyson, M., Titball, R., unpublished work), the conformation of the peptide is inconsistent with the expected binding for a proline-containing peptide. The proline side chain does not sit in the deep pocket of the enzyme: complexes with the inhibitor FK506 (see Supplementary Figure 1B online [38]) and with a cis-peptide [35] have defined this as the likely site for cis-trans isomerization. In contrast, this site is occupied by a valine side chain (Figure 2B). Furthermore, the overall conformation of the peptide is highly diverged from the expected peptide orientation, suggested by structures of rapamycin or peptide bound FKBPs (Figure 2C [34, 35, 38]). We conclude that, although BpML1 is a bona fide PPIase, this peptide binding is not representative of the binding of substrates for pre-prolyl peptide cis-trans isomerization.

However, the peptide binding is strikingly similar to the structure of human FKBP12 in complex with the transforming growth factor $\beta$ receptor I, bone morphogenic protein receptor 1B, and the activin type I receptor (A Chaikuad, I Alfano, B Shrestha, J Muniz, K Petrie, O Federov, C Phillips, S Bishop, P Mahajan, A Pike, F von Delft, C Arrowsmith, A Edwards, J Weigelt, C Bountra, S Knapp, A Bullock, unpublished work; Figure 2D; see Supplementary Figure 3 online [39]). Eukaryotic FKBPs form physiologically important complexes with other proteins, binding partner proteins to modulate their activity [40, 41], in addition to their roles in protein folding. In particular, the BpML1 peptide forms a short helix, in a similar conformation to the receptor helices that binds to hFKBP12; and each of these receptors places a leucine side chain into the deep pocket of hFKBP12, reminiscent of the valine observed in BpML1. These observations suggest that, like hFKBP12, BpML1 is likely to form complexes with other proteins in the cell in addition to its role as a PPIase. hFKBP12 masks a multiple phosphorylation peptide (the GS region) in TGF $\beta$ RI, and locks the receptor 
into an inactive conformation to ensure that there is no activation in the absence of an extracellular signal [41]. As bacteria require robust signalling networks to respond appropriately to their surroundings, similar roles in dampening signalling noise are conceivable for BpML1.

\section{X-ray and NMR structures of BPSS1823 reveal intrinsic flexibility in key active site residues}

The X-ray structure presented here diffracted to $0.91 \AA$. This resolution is high enough to reveal details of structural flexibility that cannot be observed at lower resolution. At this resolution, we can observe that $18 \%$ of the amino acid side chains (not including alanine and glycine residues) display multiple conformations. The majority of these residues are distant from the active site, in largely solvent exposed parts of the molecule. In addition to these amino acids, four alternative conformations are observed in the active site. Firstly, the pair of $\mathrm{Leu}^{-6}$ from the binding peptide and $\mathrm{Ile}^{93}$ displays correlated alternative conformers (see Supplementary Figure 4 online). More importantly, both $\mathrm{Asp}^{44}$ and $\mathrm{Tyr}^{89}$ show significant flexibility, with hydrogen bond donor and acceptor atoms moving by up to $1.5 \AA$ between the observed extremes (Figure 3). The refined positions likely represent maxima within the electron density: the two residues therefore probably sample a larger range of positions. Similar flexibility is not observed in the $0.92 \AA$ structure of unliganded hFKBP12 (see Supplementary Figure 5 online [42]), indicating that this is not an artefact of the software used. These two amino acids provide the only sources of hydrogen bond donors and acceptors in the BpML1 active site; and mutations of these residues in LpMip significantly reduce the activity of the enzyme [43]. As many proposed mechanisms of action of the enzyme involve these amino acids, this unexpected flexibility is highly suggestive of a role in catalysis. Accordingly, we examined the NMR structures of free and inhibitor-bound BpML1 to examine whether these also are consistent with a pronounced flexibility for $\mathrm{Asp}^{44}$ and $\mathrm{Tyr}^{89}$. Strikingly, the side chain of $\mathrm{Asp}^{44}$ shows a wide range of conformations (see Supplementary Figure 6 online). This is quite unusual for a residue that is apparently making a hydrogen bond interaction with the bound ligand. In contrast, the side chain of $\mathrm{Tyr}^{89}$ adopts two preferred rotamers, showing a classical alternative conformation. This contrasts with other well conserved, ligand interacting residues (e.g. Trp ${ }^{66}$ ), which show only a single conformation across 20 models consistent with the data.

This unusual flexibility of these highly conserved side chains in the active site strongly suggests a role for this flexibility in function. The side chains make hydrogen bonding interactions in at least one of their conformers, indicating that they do not lack a stable conformation. The conformations observed in the structures offer a tantalizing possible role in catalysis: superimposition of the extended prolyl peptide from the recently solved FKBPpeptide structure [35] shows that one of the $\mathrm{Tyr}^{89}$ conformers would be perfectly placed to stabilize the peptide nitrogen in an intermediate state between the cis and trans peptide. One alternative conformation of $\mathrm{Asp}^{44}$ could interact with and stabilize the peptide backbone of the substrate. These observations suggest that $\mathrm{Tyr}^{89}$ and $\mathrm{Asp}^{44}$ are natively flexible in BpML1, and that this flexibility is likely to be relevant for catalysis.

\section{The structure of BpML1 bound to cycloheximide N-ethylethanoate reveals a novel mode of inhibitor binding}

We previously showed that BpML1 binds to rapamycin, which inhibits all known FKBPs (Norville, I., Harmer, N., Harding, S., Fischer, G., Keith, K., Brown, K., Sarkar-Tyson, M., Titball, R., unpublished work). Cycloheximide, an inhibitor of eukaryotic protein synthesis, is an unrelated compound that has previously been shown to inhibit the PPIase activity of both 
hFKBP12 and LpMip (Figure 4A [44]). Cycloheximide N-ethylethanoate, which is also active against hFKBP12, inhibited the PPIase activity of BpML1 (Figure 4B): the observed $K_{\mathrm{I}}$ of 6.5 $\pm 1.0 \mu \mathrm{M}$ is similar to the $K_{\mathrm{I}}$ reported for this compound towards FKBP12 $(4.4 \mu \mathrm{M})$. As there is no extant structure of cycloheximide $\mathrm{N}$-ethylethanoate with an FKBP, we determined the structure of BpML1 bound to cycloheximide N-ethylethanoate by NMR. This structure reveals that the compound binds to the active site (Figure 4C). However, although the compound binds to most of the highly conserved side chains, the major interactions occur with the $\beta 3 b-\alpha 1$ and $\beta 4-\beta 5$ loops (Figure 4D). In fact, hydrophobic contacts are formed with residues $\mathrm{Ala}^{94}$ and Ile ${ }^{98}$, outside the core active site. No significant interactions are formed with the other sides of the catalytic bowl of the FKBP, with the highly conserved active site residues $\mathrm{Tyr}^{33}$ and $\mathrm{Phe}^{57}$ in particular having no contact with the inhibitor. In comparison, previously observed inhibitors such as FK506, rapamycin, and other synthetic inhibitors [38, $45,46]$, tend to bind more broadly across the catalytic bowl, and contact conserved active site residues across it (Figure 5). Many of these inhibitors also make extensive contacts with the same two loops as cycloheximide N-ethylethanoate.

Remarkably, the conformation of cycloheximide N-ethylethanoate mimics that of the peptide found in the BpML1 crystal structure, and the structure of hFKBP12 bound to TGF $\beta R$ (Figure 4E). Thus, cycloheximide N-ethylethanoate represents a new class of FKBP inhibitors, mimicking the binding of FKBPs to proteins to modulate activity, rather than proline-peptides as rapamycin and FK506 do.

\section{Conclusions}

Here, we report the structure of BpML1, a Mip homologue of Burkholderia pseudomallei, which was determined by X-ray crystallography and NMR in parallel. We show that this enzyme is competent in binding to peptides or the class inhibitor cycloheximide $\mathrm{N}$ ethylethanoate. Intriguingly, we show that the peptide bound in the structure is not a cisproline, but a small helical peptide that mimics TGF $\beta$ receptors bound to hFKBP12; and that cycloheximide $\mathrm{N}$-ethylethanoate binds in the active site in a way that mimics this peptide, rather than the catalytic peptides which are mimicked by other class inhibitors. These data suggest that Mips (and potentially other bacterial FKBPs), like their eukaryotic cousins, are competent to bind peptides in a helical conformation. This suggests that BpML1 and other Mips have roles in protein-protein interactions, perhaps as modulators of signalling, in addition to their chaperone functions. Consistent with this observation, deletion of BpML1 abolished B. pseudomallei showed a pleiotrophic phenotype, including loss of motility which might suggest a role in the control of the flagellar motors (Norville, I., Harmer, N., Harding, S., Fischer, G., Keith, K., Brown, K., Sarkar-Tyson, M., Titball, R., unpublished work).

The ultra-high resolution crystal structure of BpML1 revealed that two highly conserved active site side chains, $\operatorname{Asp}^{44}$ and $\mathrm{Tyr}^{89}$, show unusual flexibility. These observations were confirmed by NMR, and suggest that these side chains require flexibility, even in a ligand bound form, for performing their roles in catalysis. However, it is not clear whether this represents a general mechanism for FKBPs, or a specific case for BpML1: this enzyme is up to 6-fold more active than previously described FKBPs, and so the flexibility that we observed may be an adaptation of this enzyme to provide it with a greater rate enhancement.

The structure of the cycloheximide N-ethylethanoate complex with BpML1 suggests that this compound might provide an excellent starting point for novel drug development. The binding site for cycloheximide N-ethylethanoate, uniquely for FKBP inhibitors of known structure, is focused on the $\beta 3 b-\alpha 1$ and $\beta 4-\beta 5$ loops, and contacts residues outside the active site in the 
$\beta 4-\beta 5$ loop. This new interaction offers a greater possibility of obtaining a selective compound that would not inhibit human proteins: the $\beta 4-\beta 5$ loop, in particular, shows much greater diversity between species than the active site. Furthermore, we show here that both of these loops show considerable flexibility, increasing the likelihood of a species-specific induced fit.

We conclude that the bacterial FKBPs are likely to be a more diverse and functionally rich protein family than has previously been appreciated. In consequence of this, their validated role in the pathogenesis of several micro-organisms, and the availability of multiple inhibitors for this protein class, they show great promise for antimicrobial drug discovery. 


\section{Acknowledgements}

N.H. acknowledges the Diamond Synchrotron (Oxford, U.K.) for X-ray data collection. GV acknowledges the protein expression team at the Seattle Structural Genomics Center for Infectious Disease (SSGCID), and access to facilities at Pacific Northwest National Laboratory (Richland, WA) for NMR data collection.

\section{Funding}

K.O'S. and N.H. were supported by the University of Exeter. I.N. and M.S-T. were supported by funding from the UK Ministry of Defence. S.Z. and G.V. were supported by the National Institute for Health-National Institute of Allergy and Infectious Disease [Federal Contract HHSN272200700057C].

The protein structures reported in this manuscript have been submitted to the PDB under IDs 2KE0, 2KO7, and 2Y78. 


\section{References}

1 Wiersinga, W. J., van der Poll, T., White, N. J., Day, N. P. and Peacock, S. J. (2006) Melioidosis: insights into the pathogenicity of Burkholderia pseudomallei. Nat. Rev. Microbiol. 4, 272-282

2 White, N. (2003) Melioidosis. Lancet. 361, 1715-1722

3 Cheng, A. C. and Currie, B. J. (2005) Melioidosis: epidemiology, pathophysiology, and management. Clin. Microbiol. Rev. 18, 383-416

4 Rotz, L. D., Khan, A. S., Lillibridge, S. R., Ostroff, S. M. and Hughes, J. M. (2002)

Public health assessment of potential biological terror agents. Emerg. Infect. Dis. 8, 225-230

5 Kang, C. B., Hong, Y., Dhe-Paganon, S. and Yoon, H. S. (2008) FKBP family proteins: immunophilins with versatile biological functions. Neurosignals. 16, 318-325 6 Cianciotto, N. P., Eisenstein, B. I., Mody, C. H., Toews, G. B. and Engleberg, N. C. (1989) A Legionella pneumophila gene encoding a species-specific surface protein potentiates initiation of intracellular infection. Infect. Immun. 57, 1255-1262

7 Lundemose, A. G., Kay, J. E. and Pearce, J. H. (1993) Chlamydia trachomatis Miplike protein has peptidyl-prolyl cis/trans isomerase activity that is inhibited by FK506 and rapamycin and is implicated in initiation of chlamydial infection. Mol. Microbiol. 7, 777-783

8 Leuzzi, R., Serino, L., Scarselli, M., Savino, S., Fontana, M. R., Monaci, E., Taddei, A., Fischer, G., Rappuoli, R. and Pizza, M. (2005) Ng-MIP, a surface-exposed lipoprotein of Neisseria gonorrhoeae, has a peptidyl-prolyl cis/trans isomerase (PPIase) activity and is involved in persistence in macrophages. Mol. Microbiol. 58, 669-681

9 Moro, A., Ruiz-Cabello, F., Fernandez-Cano, A., Stock, R. P. and Gonzalez, A. (1995) Secretion by Trypanosoma cruzi of a peptidyl-prolyl cis-trans isomerase involved in cell infection. EMBO J. 14, 2483-2490

10 Helbig, J. H., Konig, B., Knospe, H., Bubert, B., Yu, C., Luck, C. P., RiboldiTunnicliffe, A., Hilgenfeld, R., Jacobs, E., Hacker, J. and Fischer, G. (2003) The PPIase active site of Legionella pneumophila Mip protein is involved in the infection of eukaryotic host cells. Biol. Chem. 384, 125-137

11 Wagner, C., Khan, A. S., Kamphausen, T., Schmausser, B., Unal, C., Lorenz, U., Fischer, G., Hacker, J. and Steinert, M. (2007) Collagen binding protein Mip enables Legionella pneumophila to transmigrate through a barrier of NCI-H292 lung epithelial cells and extracellular matrix. Cell. Microbiol. 9, 450-462

12 Zang, N., Tang, D. J., Wei, M. L., He, Y. Q., Chen, B., Feng, J. X., Xu, J., Gan, Y. Q., Jiang, B. L. and Tang, J. L. (2007) Requirement of a mip-like gene for virulence in the phytopathogenic bacterium Xanthomonas campestris pv. campestris. Mol. Plant. Microbe. Interact. 20, 21-30

13 Studier, F. W. (2005) Protein production by auto-induction in high-density shaking cultures. Prot. Expres. Purif. 41, 207-234

14 Leslie, A. (1992) Recent changes to the MOSFLM package for processing film and image plate data. Joint CCP4 ESF-EAMCB Newslett. Prot. Crystallogr. 26

15 Evans, P. R. (2005) Scaling and assessment of data quality. Acta Crystallogr. D. D62, $72-82$

16 Stein, N. (2008) CHAINSAW: a program for mutating pdb files used as templates in molecular replacement. J. Appl. Crystallogr. 41, 641-643

17 McCoy, A. J., Grosse-Kunstleve, R. W., Adams, P. D., Winn, M. D., Storoni, L. C. and Read, R. J. (2007) Phaser crystallographic software. J. Appl. Crystallogr. 40, 658-674 18 Emsley, P., Lohkamp, B., Scott, W. G. and Cowtan, K. (2010) Features and development of Coot. Acta Crystallogr. D. D66, 486-501 
19 Adams, P. D., Grosse-Kunstleve, R. W., Hung, L.-W., Ioerger, T. R., McCoy, A. J., Moriarty, N. W., Read, R. J., Sacchettini, J. C., Sauter, N. K. and Terwilliger, T. C. (2002) PHENIX: building new software for automated crystallographic structure determination. Acta Crystallogr. D. D58, 1948-1954

20 Davis, I. W., Leaver-Fay, A., Chen, V. B., Block, J. N., Kapral, G. J., Wang, X., Murray, L. W., Arendall III, B., Snoeyink, J., Richardson, J. S. and Richardson, D. C. (2007) MolProbity: all-atom contacts and structure validation for proteins and nucleic acids. Nucleic Acids Res. 35, W375-W383

21 Kay, L. E., Xu, G. Y., Singer, A. U., Muhandiram, D. R. and Formankay, J. D. (1993) A Gradient-Enhanced Hcch Tocsy Experiment for Recording Side-Chain H-1 and C-13 Correlations in $\mathrm{H}_{2} \mathrm{O}$ Samples of Proteins. J. Magn. Reson. Ser. B. 101, 333-337 22 Muhandiram, D. R., Xu, G. Y. and Kay, L. E. (1993) An Enhanced-Sensitivity Pure Absorption Gradient 4d N-15, C-13-Edited Noesy Experiment. J. Biomol. Nmr. 3, 463-470 23 Yamazaki, T., Lee, W., Revington, M., Mattiello, D. L., Dahlquist, F. W., Arrowsmith, C. H. and Kay, L. E. (1994) An Hnca Pulse Scheme for the Backbone Assignment of N-15,C-13,H-2-Labeled Proteins - Application to a 37-Kda Trp Repressor DNA Complex. J. Am. Chem. Soc. 116, 6464-6465

24 Muhandiram, D. R. and Kay, L. E. (1994) Gradient-Enhanced Triple-Resonance 3Dimensional Nmr Experiments with Improved Sensitivity. J. Magn. Reson. Ser. B. 103, 203216

25 Peterson, R. D., Theimer, C. A., Wu, H. H. and Feigon, J. (2004) New applications of 2D filtered/edited NOESY for assignment and structure elucidation of RNA and RNA-protein complexes. J. Biomol. Nmr. 28, 59-67

26 Zwahlen, C., Legault, P., Vincent, S. J. F., Greenblatt, J., Konrat, R. and Kay, L. E. (1997) Methods for measurement of intermolecular NOEs by multinuclear NMR spectroscopy: Application to a bacteriophage lambda N-peptide/boxB RNA complex. J. Am. Chem. Soc. 119, 6711-6721

27 Delaglio, F., Grzesiek, S., Vuister, G. W., Zhu, G., Pfeifer, J. and Bax, A. (1995) Nmrpipe - a Multidimensional Spectral Processing System Based on Unix Pipes. J. Biomol. Nmr. 6, 277-293

28 Vranken, W. F., Boucher, W., Stevens, T. J., Fogh, R. H., Pajon, A., Llinas, P., Ulrich, E. L., Markley, J. L., Ionides, J. and Laue, E. D. (2005) The CCPN data model for NMR spectroscopy: Development of a software pipeline. Proteins. 59, 687-696

29 Güntert, P. (2003) Automated NMR protein structure calculation. Prog. Nucl. Mag. Res. Sp. 43, 105-125

30 Herrmann, T., Güntert, P. and Wuthrich, K. (2002) Protein NMR structure determination with automated NOE assignment using the new software CANDID and the torsion angle dynamics algorithm DYANA. J. Mol. Biol. 319, 209-227

31 Fischer, G., Bang, H. and Mech, C. (1984) Detection of Enzyme Catalysis for CisTrans-Isomerization of Peptide-Bonds Using Proline-Containing Peptides as Substrates. Biomed. Biochim. Acta. 43, 1101-1111

32 Kullertz, G., Luthe, S. and Fischer, G. (1998) Semiautomated microtiter plate assay for monitoring peptidylprolyl cis/trans isomerase activity in normal and pathological human sera. Clin. Chem. 44, 502-508

33 Williams, J. W. and Morrison, J. F. (1979) The kinetics of reversible tight-binding inhibition. Methods Enzymol. 63, 437-467

34 Ceymann, A., Horstmann, M., Ehses, P., Schweimer, K., Paschke, A. K., Steinert, M. and Faber, C. (2008) Solution structure of the Legionella pneumophila Mip-rapamycin complex. BMC Struct. Biol. 8, 17 
35 Löw, C., Neumann, P., Tidow, H., Weininger, U., Haupt, C., Friedrich-Epler, B., Scholz, C., Stubbs, M. T. and Balbach, J. (2010) Crystal structure determination and functional characterization of the metallochaperone SlyD from Thermus thermophilus. J. Mol. Biol. 398, 375-390

36 Pereira, P. J., Vega, M. C., Gonzalez-Rey, E., Fernandez-Carazo, R., Macedo-Ribeiro, S., Gomis-Ruth, F. X., Gonzalez, A. and Coll, M. (2002) Trypanosoma cruzi macrophage infectivity potentiator has a rotamase core and a highly exposed alpha-helix. EMBO Rep. 3, $88-94$

37 Riboldi-Tunnicliffe, A., Konig, B., Jessen, S., Weiss, M. S., Rahfeld, J., Hacker, J., Fischer, G. and Hilgenfeld, R. (2001) Crystal structure of Mip, a prolylisomerase from Legionella pneumophila. Nat. Struct. Biol. 8, 779-783

38 Wilson, K. P., Yamashita, M. M., Sintchak, M. D., Rotstein, S. H., Murcko, M. A., Boger, J., Thomson, J. A., Fitzgibbon, M. J., Black, J. R. and Navia, M. A. (1995)

Comparative X-ray structures of the major binding protein for the immunosuppressant FK506 (tacrolimus) in unliganded form and in complex with FK506 and rapamycin. Acta Crystallogr. D. 51, 511-521

39 Huse, M., Chen, Y. G., Massague, J. and Kuriyan, J. (1999) Crystal structure of the cytoplasmic domain of the type I TGF beta receptor in complex with FKBP12. Cell. 96, 425436

40 Chelu, M. G., Danila, C. I., Gilman, C. P. and Hamilton, S. L. (2004) Regulation of ryanodine receptors by FK506 binding proteins. Trends Cardiovas. Med. 14, 227-234

41 Huse, M., Muir, T. W., Xu, L., Chen, Y. G., Kuriyan, J. and Massague, J. (2001) The TGF beta receptor activation process: an inhibitor- to substrate-binding switch. Mol. Cell. 8, 671-682

42 Szep, S., Park, S., Boder, E. T., Van Duyne, G. D. and Saven, J. G. (2009) Structural coupling between FKBP12 and buried water. Proteins. 74, 603-611

43 Wintermeyer, E., Ludwig, B., Steinert, M., Schmidt, B., Fischer, G. and Hacker, J. (1995) Influence of site specifically altered Mip proteins on intracellular survival of Legionella pneumophila in eukaryotic cells. Infect. Immun. 63, 4576-4583

44 Christner, C., Wyrwa, R., Marsch, S., Kullertz, G., Thiericke, R., Grabley, S., Schumann, D. and Fischer, G. (1999) Synthesis and cytotoxic evaluation of cycloheximide derivatives as potential inhibitors of FKBP12 with neuroregenerative properties. J. Med. Chem. 42, 3615-3622

45 Sich, C., Improta, S., Cowley, D. J., Guenet, C., Merly, J. P., Teufel, M. and Saudek, V. (2000) Solution structure of a neurotrophic ligand bound to FKBP12 and its effects on protein dynamics. Eur. J. Biochem. 267, 5342-5355

46 Sun, F., Li, P., Ding, Y., Wang, L., Bartlam, M., Shu, C., Shen, B., Jiang, H., Li, S. and Rao, Z. (2003) Design and structure-based study of new potential FKBP12 inhibitors. Biophys. J. 85, 3194-3201

47 Cornilescu, G., Delaglio, F. and Bax, A. (1999) Protein backbone angle restraints from searching a database for chemical shift and sequence homology. J. Biomol. Nmr. 13, 289-302 48 Fulton, K. F., Jackson, S. E. and Buckle, A. M. (2003) Energetic and structural analysis of the role of tryptophan 59 in FKBP12. Biochemistry. 42, 2364-2372

49 Baker, N., Sept, D., Joseph, S., Holst, M. and McCammon, J. (2001) Electrostatics of nanosystems: application to microtubules and the ribosome. Proc. Natl. Acad. Sci. U.S.A. 98, 10037-10041

50 Dolinsky, T. J., Czodrowski, P., Li, H., Nielsen, J. E., Jensen, J. H., Klebe, G. and Baker, N. A. (2007) PDB2PQR: expanding and upgrading automated preparation of biomolecular structures for molecular simulations. Nucleic Acids Res. 35, W522-525 
Table 1: Data collection and refinement statistics

\begin{tabular}{ll}
\hline & BpML1 (PDB ID: 2Y78) \\
\hline $\begin{array}{l}\text { Data collection } \\
\text { Space group }\end{array}$ & $P 4{ }_{3} 2{ }_{1} 2$ \\
Cell dimensions & \\
$\quad a, b, c(\AA)$ & $54.68,54.68,119.2$ \\
$\quad \alpha, \beta, \gamma\left({ }^{\circ}\right)$ & $90,90,90$ \\
Resolution $(\AA)$ & $40.3-0.91(0.96-0.91)^{*}$ \\
$R_{\text {merge }}$ & $0.080(0.398)$ \\
$I / \sigma I$ & $13.8(2.2)$ \\
Completeness $(\%)$ & $98.3(88.2)$ \\
Redundancy & $5.9(2.7)$ \\
& \\
Refinement & \\
Resolution $(\AA)$ & $40.3-0.91$ \\
No. reflections & 125,244 \\
$R_{\text {work }} / R_{\text {free }}$ & $0.109 / 0.116$ \\
No. atoms & \\
$\quad$ Protein & 2032 \\
Ligand/ion & 58 \\
$\quad$ Water & 275 \\
$B$-factors & 12.7 \\
Protein & 27.2 \\
Ligand/ion & 24.2 \\
Water & \\
R.m.s. deviations & 0.014 \\
Bond lengths $(\AA)$ & 1.586 \\
$\quad$ Bond angles $\left({ }^{\circ}\right)$ & \\
\hline All & \\
\hline
\end{tabular}

${ }^{\dagger}$ All data were collected from one crystal. High and low resolution passes were merged.

*Values in parentheses are for highest-resolution shell. 
Table 2: NMR and refinement statistics

\begin{tabular}{|c|c|c|}
\hline & $\begin{array}{c}\text { Apo- } \\
\text { BpML1 } \\
\text { (PDB ID: } \\
\text { 2KE0) }\end{array}$ & $\begin{array}{c}\text { BpML1-CNE } \\
\text { complex (PDB ID: } \\
\text { 2KO7) }\end{array}$ \\
\hline \multicolumn{3}{|l|}{ NMR distance and dihedral } \\
\hline \multicolumn{3}{|l|}{ Constraints } \\
\hline \multicolumn{3}{|l|}{ Structure constraints } \\
\hline \multicolumn{3}{|l|}{ Distance constraints $^{\mathrm{a}}$} \\
\hline Total meaningful NOE & 1792 & 1757 \\
\hline \multicolumn{3}{|l|}{ Restraints } \\
\hline Short range $(|i-j|<=1)$ & 777 & 808 \\
\hline Medium range $(1<|i-j|<5)$ & 216 & 205 \\
\hline Long range $(|i-j|>=5)$ & 799 & 744 \\
\hline Hydrogen bond restraints & 78 & 92 \\
\hline Dihedral angle restraints $(\phi, \psi)^{\mathrm{b}}$ & 134 & 136 \\
\hline Total number of constraints & 2004 & 1985 \\
\hline Constraints per residue & 17.1 & 16.8 \\
\hline Inter molecular NOE restraints & & 39 \\
\hline \multicolumn{3}{|l|}{ Residual constraint violations $^{c}$} \\
\hline Cyana target function & 0.84 & 1.54 \\
\hline Distance violations $(>0.2 \AA)$ & 3 & 4 \\
\hline Torsion angles $\left(>5.0^{\circ}\right)$ & 0 & 0 \\
\hline \multicolumn{3}{|l|}{ Ramachandran statistics $(\%)^{\mathrm{d}}$} \\
\hline Favoured & 80.8 & 80.7 \\
\hline Additionally allowed & 19.0 & 19.0 \\
\hline Generously allowed & 0.1 & 0.3 \\
\hline Disallowed & 0.0 & 0.0 \\
\hline \multicolumn{3}{|l|}{ Average RMSD to the mean $(\AA)^{\mathrm{e}}$} \\
\hline Backbone & $0.39 \pm 0.05$ & $0.52 \pm 0.15$ \\
\hline Heavy atom & $0.89 \pm 0.08$ & $0.98 \pm 0.15$ \\
\hline Ligand heavy atom & & $1.11 \pm 0.14$ \\
\hline $\begin{array}{l}{ }^{\mathrm{a}} \text { Final } 20 / 100 \text { structures selected ba } \\
\text { b Dihedral angle restraints derived } \mathrm{f} \\
{ }^{\mathrm{c}} \text { Maximum violation shown in pare } \\
{ }^{\mathrm{d}} \text { Ramachandran analysis was perfo } \\
\text { e }\end{array}$ & $\begin{array}{l}\mathrm{d} \text { on the low } \\
\mathrm{m} \text { TALOS [ } \\
\text { heses. } \\
\text { hed with PRC } \\
\text { he protein w }\end{array}$ & CYANA target func \\
\hline
\end{tabular}




\section{Figure Legends}

Figure 1: Structures of B. pseudomallei Mip (BpML1).

(a) Cartoon of the $0.91 \AA$ structure of BpML1 (left, green; PDB ID: 2Y78) compared with hFKBP12 (right, cyan; PDB ID: 2DG3 [48]). The overall fold is identical, with BpML1 containing all of the secondary structural elements, and an additional $\mathrm{N}$-terminal $\beta$-strand (arrowed). (b) BpML1 shows a nearly identical active site conformation to LpMip (purple; PDB ID: 1FD9). All of the most conserved side chains are identical in conformation, except for $\mathrm{Phe}^{46}$ (arrow), which is replaced functionally in LpMip with a neighbouring Phe. (c): The NMR structure of BpML1 confirms the crystallographic data and identifies flexibility within the active site. Left: Cartoon of the NMR structure of BpML1 bound to ligand (yellow; PDB ID: 2KO7) shows a very similar architecture to the crystal structure (a). Right: the comparison of bound (yellow) and apo (pink: PDB ID: 2KE0) shows significant conformational flexibility in the ligand-binding $\beta 3$ a strand (arrow) and the $\beta 4-\beta 5$ loop (arrowhead). Both structures shown are representative for an ensemble of 20 structures consistent with the data.

Figure 2: BpML1 binding to thrombin recognition peptide suggests that the protein is involved in protein-protein interactions.

(a) Analysis of crystal packing in the X-ray structure of BpML1 (PDB ID: 2Y78) shows a helical peptide binding to the active site. Cartoon is shown of BpML1 (green) and the peptide derived from cloning (orange). (b) The peptide binds with a valine side chain (black arrow) in the heart of the enzyme active site. The peptide is shown in orange sticks, with BpML1 as green cartoon. The grey arrow indicates the direction of the peptide, $\mathrm{N}$ to $\mathrm{C}$ terminus. (c) The peptide binding is unlikely to represent a substrate for the PPIase activity. Comparison with the structure of a cis-prolyl peptide bound to SlyD from T. thermophilus (left, yellow; PDB ID: 3LUO) and FK-506 bound to hFKBP12 (right, cyan; FK-506 pseudopeptide shown in yellow; PDB ID: 1FKJ) shows that a bona fide cis-proline peptide lying in the opposite orientation (grey arrows); and that a prolyl or pseudoprolyl side chain occupies the active site (black arrowheads). (d) BpML1 peptide binding mimics hFKBP12 binding to TGF $\beta R I$. Left: BpML1 (green) binding to peptide (white sticks). Right: hFKBP12 (cyan) binding to a helix from TGF $\beta R I$ (pink sticks). The side chains binding to the heart of the active sites are coloured yellow. Note the similarity of the positions of the carbonyls in the red boxes. Six peptide main chain atoms making direct or through single water-mediated hydrogen bonds with BpML1 are shown as spheres on top of the sticks, and are further indicated by black arrowheads.

Figure 3: The ultra-high resolution X-ray structure of BpML1 (PDB ID: 2Y78) shows multiple conformers of key ligand-binding residues.

(a) $\mathrm{Asp}^{44}$, which accepts a key hydrogen bond from the bound peptide backbone, shows two conformers for the side chain. The distance between the two conformations for $\mathrm{O}_{\delta 2}$ is $0.9 \AA$. The higher occupancy conformation makes an additional hydrogen bond to the bound peptide backbone, while the other makes two additional hydrogen bonds to other residues of BpML1. (b) $\mathrm{Tyr}^{89}$, the only hydrogen bond donor in the active site pocket, also shows a range of conformations. The distance between the $\mathrm{O}_{\eta}$ atoms is $1.5 \AA$. One conformation is consistent with a direct $(3.0 \AA$ ) hydrogen bond to the peptide, while the other makes a water-mediated hydrogen bond to the peptide. Lower occupancy forms are indicated by a black arrow. (c) Representative electron density for a multiple conformer residue (Tyr ${ }^{89}$, left) and the peptide binding to the active site (right). Colours: BpML1, yellow; binding peptide, orange; multiple conformation at lower occupancy, cyan; $\mathrm{mF}_{\mathrm{o}}-\mathrm{DF}_{\mathrm{c}}$ simulated annealing omit map (side chains 
truncated to $\mathrm{C}_{\beta}$ ) contoured at $3 \sigma$, green. $2 \mathrm{mF}_{\mathrm{o}}-\mathrm{DF}_{\mathrm{c}}$ map for the final structure contoured at $1 \sigma$, blue.

Figure 4: Cycloheximide N-ethylethanoate identifies a novel binding mode for FKBP inhibitors.

(a) Structures of cycloheximide (left) and its derivative cycloheximide N-ethylethanoate. (b) Inhibition of BpML1 by cycloheximide N-ethylethanoate: cycloheximide N-ethylethanoate inhibited the PPIase activity of BpML1 in a dose dependent manner, showing a $K_{\mathrm{I}}$ of $6.5 \pm 1.0$ $\mu \mathrm{M}$. All experiments were performed in triplicate, and standard errors of the mean are shown. The solid line shows the inhibition curve for fit values of the inhibition parameters. (c) Overview of the NMR structure of cycloheximide N-ethylethanoate (orange spheres) bound to BpML1 (PDB ID: 2KO7). Left: BpML1 shown as yellow cartoon. Right: Surface of BpML1 shown, coloured by charge density (red: negative; blue: positive). Charge density was calculated using APBS [49] and PDB2PQR [50]. (d) Close-up view of active site residues binding to cycloheximide N-ethylethanoate. Residues within $3.5 \AA$ of cycloheximide Nethylethanoate are shown as sticks. Left: BpML1 shown as cartoon, cycloheximide Nethylethanoate shown as sticks. The hydrogen bond between the cycloheximide Nethylethanoate hydroxyl and $\mathrm{Asp}^{44}$ is especially noteworthy; and that some functional groups of cycloheximide N-ethylethanoate (arrowed) do not make any interactions with the protein, and could be altered. Right: BpML1 is shown as semi-transparent surface, cycloheximide Nethylethanoate as spheres. cycloheximide N-ethylethanoate shows good shape complementarity to BpML1, consistent with its micromolar inhibitory activity. (e) Cycloheximide N-ethylethanoate mimics the binding of peptides to FKBPs. Left: cycloheximide $\mathrm{N}$-ethylethanoate (light orange sticks; atoms mimicking the peptide coloured bolder and shown thicker) bound to BpMip (yellow cartoon; PDB ID: 2KO7). Centre: peptide (white sticks; atoms equivalent to those highlighted left shown light grey and thicker) bound to BpMip (green cartoon; PDB ID: 2Y78). Right: helix from TGF $\beta$ RI (pink sticks) binding to hFKBP12 (cyan cartoon; PDB ID: 1B6C). The relevant portions of each peptide are highlighted with black arrowheads, whilst hydrophobic side chains/cycloheximide Nethylethanoate atoms binding to the protein are highlighted with open arrowheads. The black arrow indicates a section of cycloheximide $\mathrm{N}$-ethylethanoate that binds to a BpML1 pocket that is not accessed by the peptides. Note how the cycloheximide N-ethylethanoate molecule binds closer to the $\beta 4-\beta 5$ loop than the peptides.

Figure 5: Comparison of the binding of FKBP inhibitors to FKBPs indicates that cycloheximide $\mathrm{N}$-ethylethanoate represents a novel class of inhibitor.

Inhibitors are shown as all heavy atom spherical models, with carbon coloured yellow, oxygen red, and nitrogen blue; proteins are shown as white, semi-transparent surfaces, with cartoon beneath. Cartoon colours: BpMip, yellow; FKBP12, cyan. The nine most conserved active site residues of FKBPs are shown as sticks, with carbon coloured as for the main chain, and other atoms as above. Side chains $\mathrm{Tyr}^{33}$ and $\mathrm{Phe}^{57}$ of BpMip do not contact the inhibitor, and are highlighted with carbon coloured orange and with black arrows; equivalent FKBP12 residues are highlighted with carbon coloured orange and with black arrowheads. (a) BpMip binding to cycloheximide N-ethylethanoate (this work; PDB ID: 2KO7). (b) FKBP12 binding to rapamycin (PDB ID: 2DG3). (c) FKBP12 binding to FK-506 (PDB ID: 1FKJ). (d) FKBP12 binding to the neurotrophic ligand GPI-1046 (PDB ID: 1F40). (e, f, g) FKBP12 binding to synthetic, high affinity ligands (PDB IDs: 1FKG, 1FKI, 1J4R). (h) FKBP12 binding to a synthetic, dimeric ligand (PDB ID: 1A7X). Note that cycloheximide N-ethylethanoate occupies a distinct area of the protein, and in particular makes no interaction with the $\beta 3 \mathrm{~b}$ strand (black dashed arrow). 

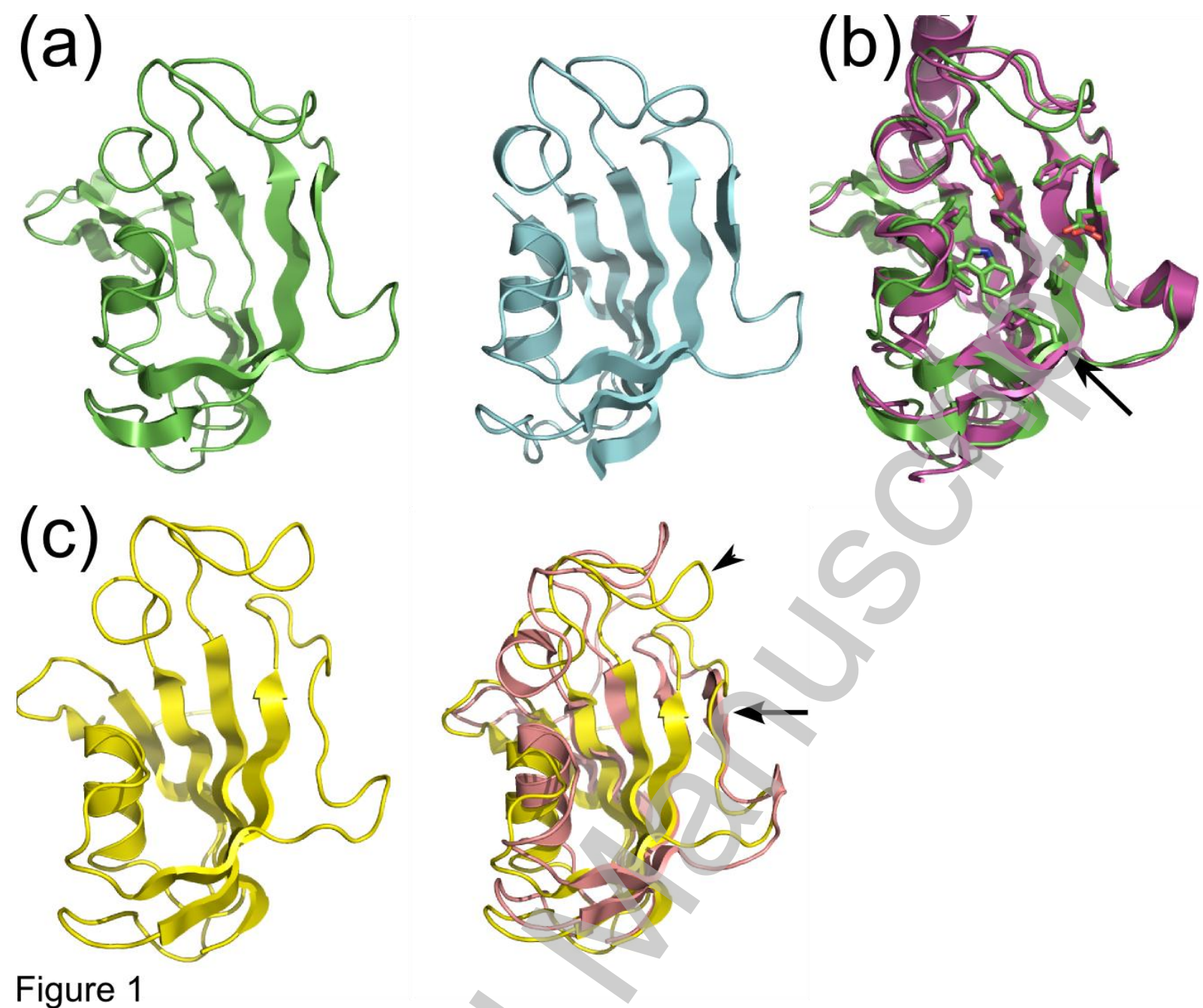

Figure 1 
(a)

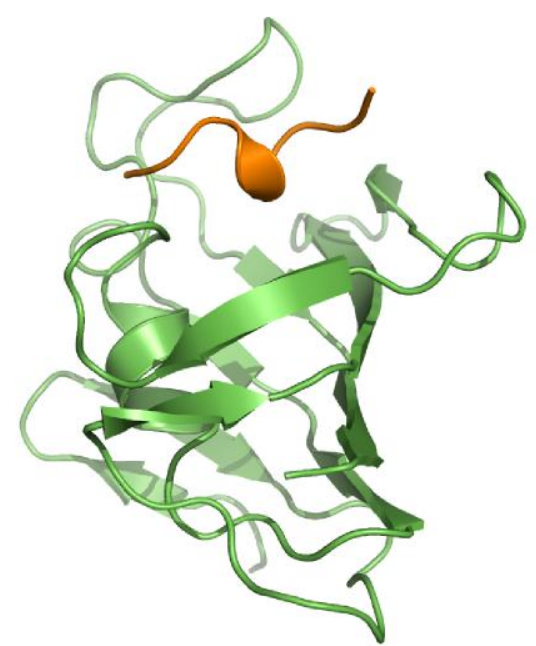

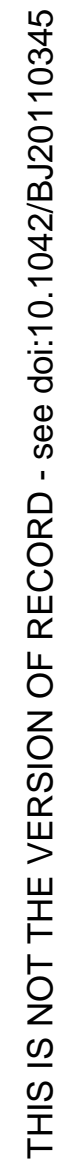

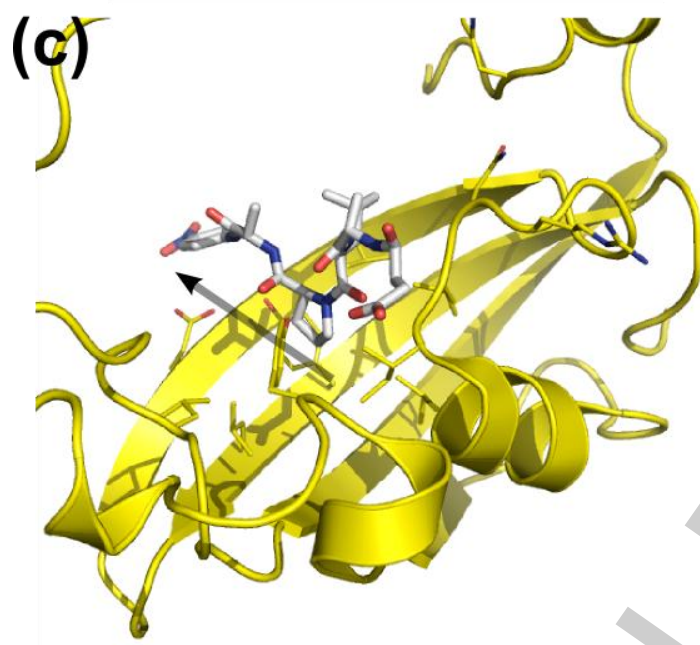

(b)

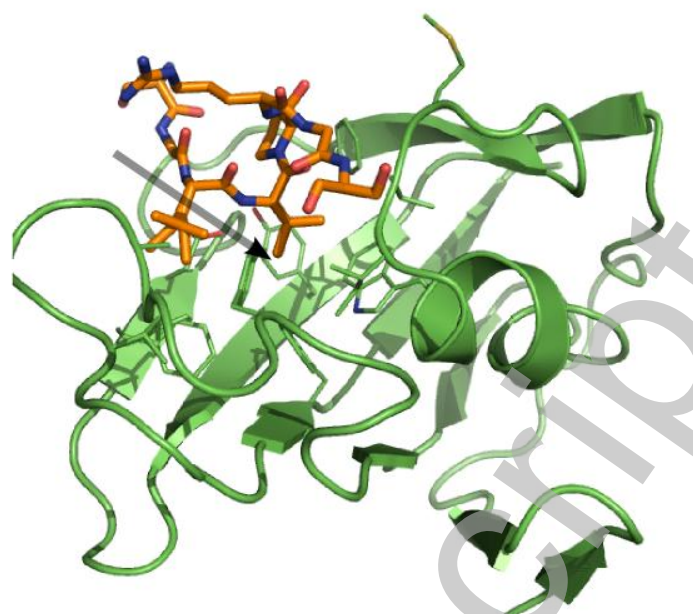




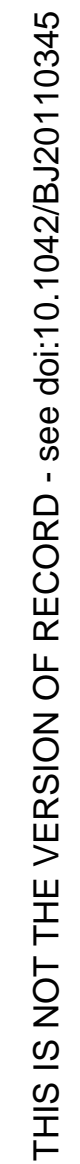

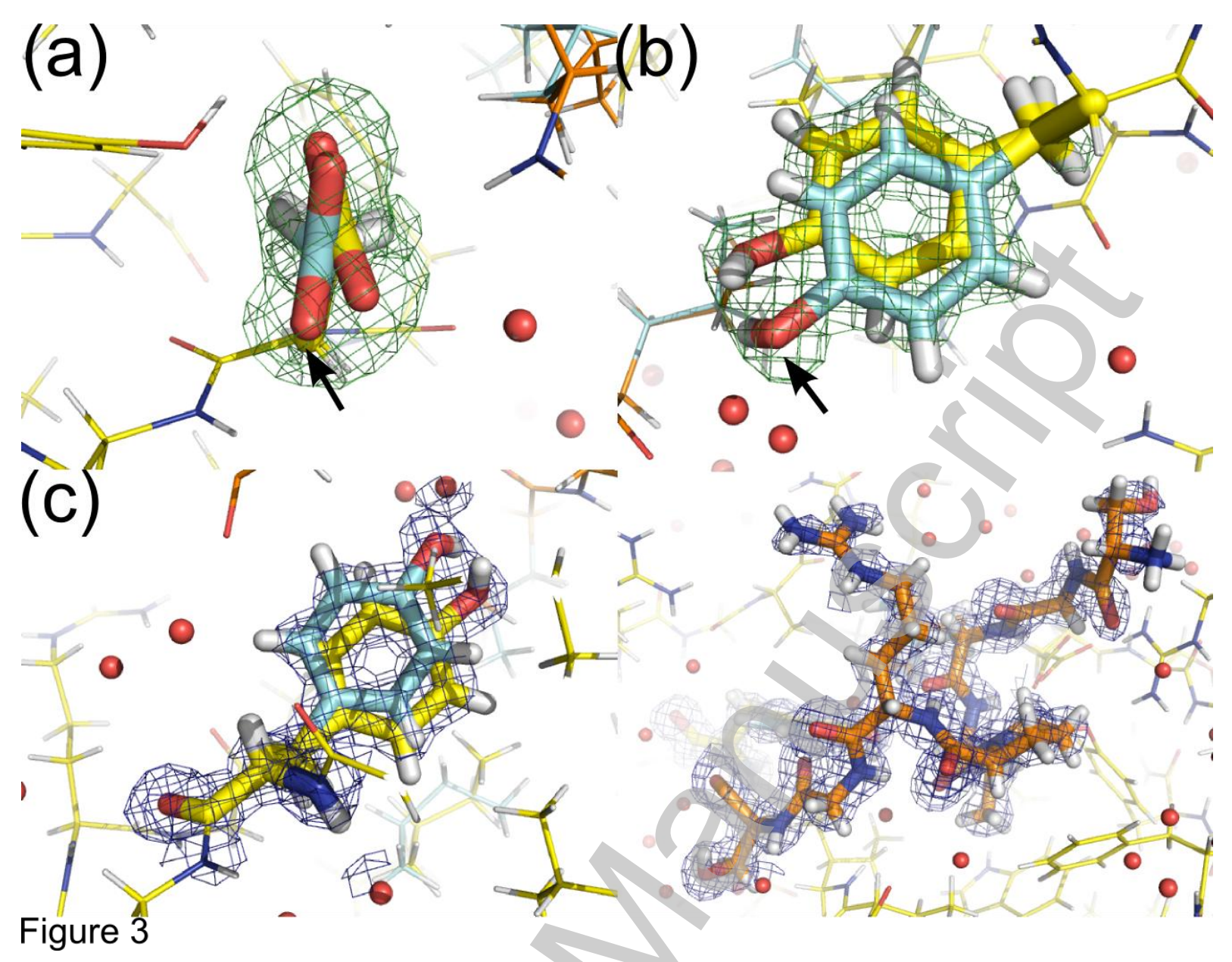


(a)<smiles>C[C@@H]1C[C@@H]([C@H](O)CC2CC(=O)NC(=O)C2)C(=O)[C@H](C)C1</smiles>

(c)

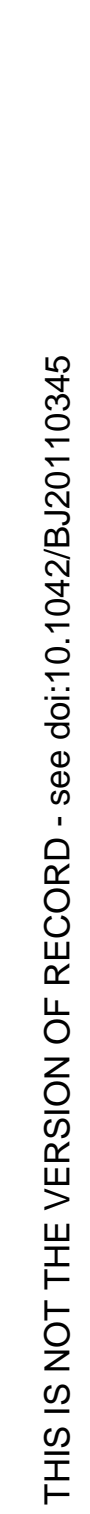

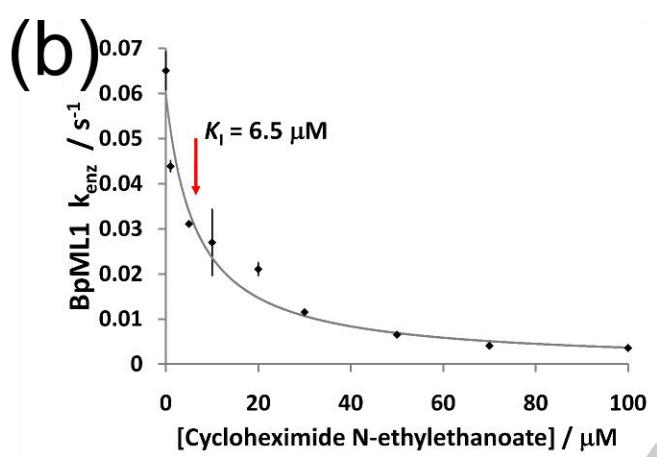

Licenced copy. Copying is not permitted, except with prior permission and as allowed by law. (C) 2011 The Authors Journal compilation (c) 2011 Portland Press Limited 

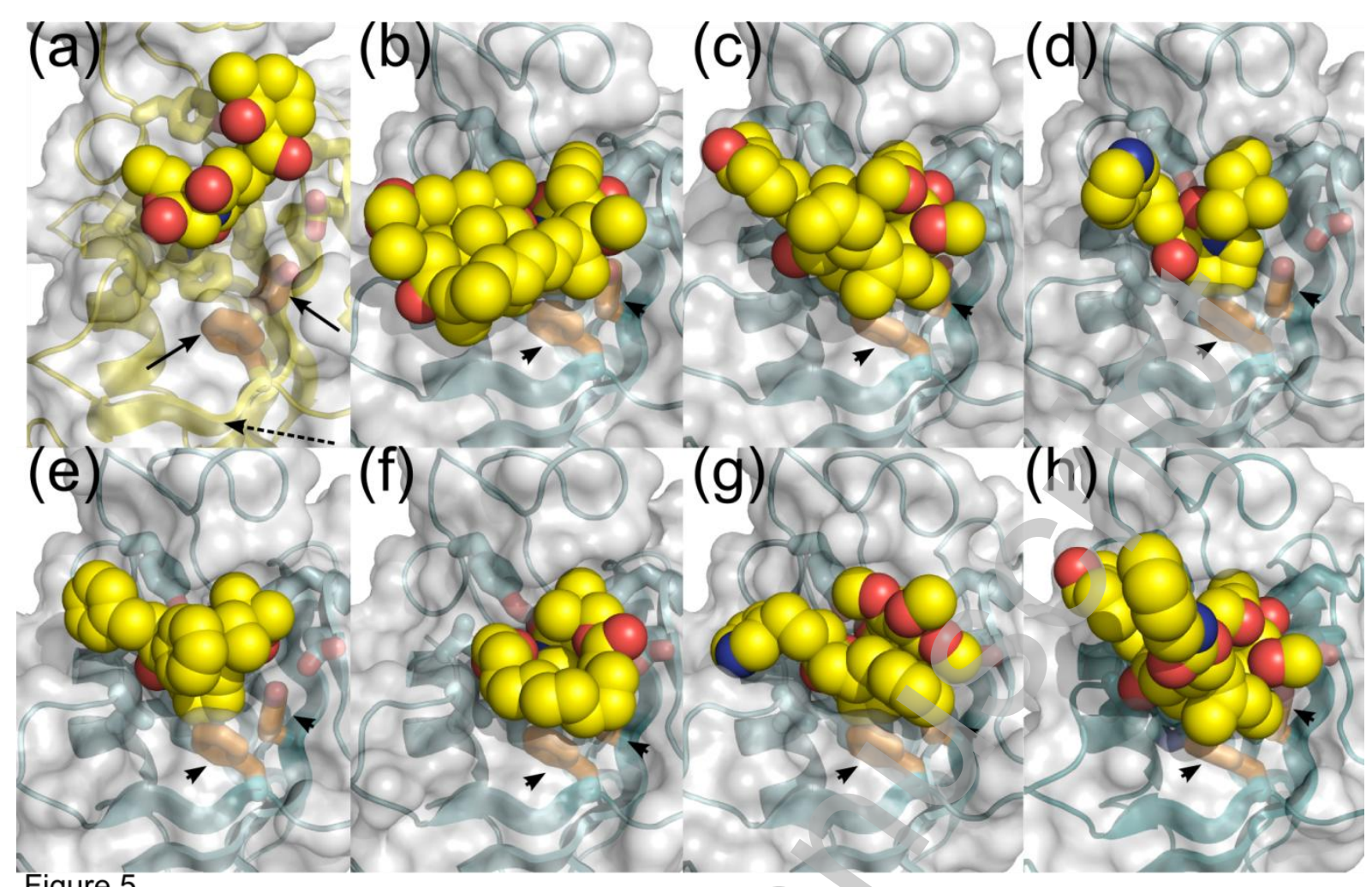

Figure 5

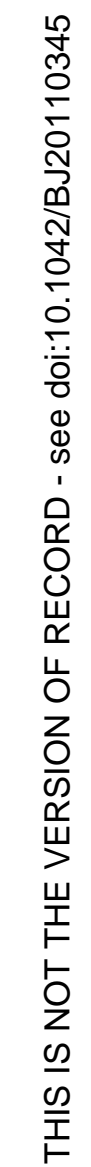

